\title{
The goldfish conditioned withdrawal preparation: Effects of some basic methodological variables
}

\author{
Peter B. Barela ${ }^{1}$
}

Published online: 16 May 2015

(C) Psychonomic Society, Inc. 2015

\begin{abstract}
Barela (Psychological Reports, 110: 173-186, 2012) presented a novel preparation for the experimental study of classical fear conditioning in goldfish, and successfully employed it in the demonstration of several basic conditioning principles. The present research addressed some basic methodological questions using a modified version of this preparation. Results indicated that the modification made to the visual CS increased its efficacy, and that conditioning to this stimulus was directly related to the number of CS-US trials given, US intensity, and intertrial interval. In addition, conditioning was detected with interstimulus intervals both shorter and longer than those used previously, as well as with an auditory CS. Methodological implications for the future employment of this preparation are discussed.
\end{abstract}

Keywords Goldfish · Classical conditioning · Fear ·

Withdrawal $\cdot$ Animal models $\cdot$ Methods

Due to the high costs, extensive facilities, and numerous governmental regulations associated with the use of laboratory rodents, a strong need has been expressed for an alternative subject that can be used in courses on animal learning (Miskovsky, Becker, Hilker, \& Abramson, 2010; Phelps, 2014; Varnon \& Abramson, 2013). In response to this need, Miskovsky et al. and Phelps have made advances in the development of methods for the study of operant conditioning in goldfish, and Barela (2012) has developed a novel preparation for the study of classical fear conditioning in these animals that involves the measurement of withdrawal from a light-conditioned stimulus (CS)

Peter B. Barela

peter.barela@yahoo.com

1 Henderson, CO, USA that has previously been paired with an aversive shockunconditioned stimulus (US). The unique features of this preparation are that it is easy for the novice to construct and operate, by hand, with materials that are technically unsophisticated, readily available, and inexpensive (less than $\$ 200$ for materials, and less than 20 apiece for feeder goldfish). Importantly, Barela showed how this preparation can be used to demonstrate several basic conditioning principles, namely, excitatory conditioning, extinction, contextual conditioning, blocking, a conditioned inhibition discrimination, and latent inhibition.

In addition to its being of use in the classroom, Barela (2012) suggested that this preparation (or some variation thereof) might also serve animal learning researchers as a viable, low-cost alternative to preparations that employ rodents, the current subject of choice in this area. Two lines of experimental research provided the basis for this suggestion: (1) studies showing that rats and goldfish obey the same laws of learning, and (2) studies indicating that the homologous brain structures in these animals subserve similar cognitive functions. With 10-year budget caps and sequester cuts (signed into law under the Budget Control Act of 2011) scheduled to go into full effect in 2016, and funding for basic research in the behavioral sciences traditionally considered a relatively low priority by the U.S. Congress (see, e.g., Skedsvold, 2015), it would be prudent for researchers to seriously consider the feasibility of using lower cost alternatives to traditional conditioning preparations.

After his 2012 report, Barela attempted to use that preparation in a professional research capacity (i.e., to address certain theoretical questions), but immediately encountered technical and methodological difficulties which led him to make two important observations. The first was that the efficacy of this preparation needed to be improved. Hence, the following modifications were made. First, the shock-US was increased from $7 \mathrm{~V}$ to $10 \mathrm{~V}$, which resulted in a more consistent and 
vigorous thrashing unconditioned response (UR), without causing any apparent physical damage to the goldfish (i.e., outside the conditioning chamber, they were indistinguishable from those that were experimentally naïve). ${ }^{1}$ Second, the two red Christmas light-CSs were replaced by two relatively small, red pilot lights (similar to those used in standard Skinner boxes), each positioned against a black background, a change which appeared to increase the salience of the CS. Third, the size of the conditioning chamber was increased by $45 \%$ to allow for the use of larger goldfish, which are oftentimes healthier than smaller ones, especially when purchased from retail pet stores. Fourth, Barela (unpublished observations) had noticed that, upon being placed in the conditioning box for the CS test, goldfish will sometimes freeze (defined as the complete lack of body, head, and tail-fin movement) for a short period of time (usually less than $1 \mathrm{~min}$, but occasionally longer), and that this behavior can interfere with performance of the conditioned withdrawal response. Henceforth, if the goldfish happened to be freezing when the CS was to be presented, the test was delayed until this behavior ceased (usually no more than $5 \mathrm{~min}$ into the session). Finally, a buzzer was positioned directly under each light-CS to determine whether this preparation can also be used to measure conditioning to an auditory CS, a feature that would be of much use to researchers in this area.

The second observation made by Barela was that, because of the novelty of this preparation, having some basic parametric data available would greatly expedite the selection of optimal experimental methods for detecting various treatment effects. As all students of learning know, careful consideration must be given to the experimental methods employed because many of the variables involved (e.g., number of trials, intertrial interval [ITI], CS modality, etc.) can have substantial effects on learning and/or performance (see, e.g., Holland, 1977; Mackintosh, 1974, pp. 8-77). Therefore, the following experiments were conducted to provide some of these parametric data and to determine whether some of the above modifications actually did increase the efficacy of this preparation. In addition, the data obtained were subjected to power analyses to allow future employers of this preparation to estimate the minimum number of subjects needed to detect small, medium, and large treatment effects (see Cohen, 1988). The results have important implications for this preparation's use, both in the classroom and in professional research laboratories.

\footnotetext{
${ }^{1}$ Although the power supply used by Barela (2012) to produce the shock-US indicated that its output was $6 \mathrm{~V}$, a subsequent independent measurement revealed that it was actually closer to $7 \mathrm{~V}$.
}

\section{Experiment 1A}

One methodological variable that can have major effects on classical conditioning is, of course, the number of CS-US trials that are given. When individual data are averaged to represent group behavior, the functional relationship between these variables has been found to be direct and negativelyaccelerated (for a review, see Gallistel, Fairhurst, \& Balsam, 2004). Using the modified version of the goldfish conditioned withdrawal preparation, Barela (unpublished observations) found that 10 trials given with 3-min ITIs produced a level of conditioning that was at, or at least very much near, asymptote. The present experiment was conducted to chart the growth of conditioning that occurs to the light-CS in this preparation as the number of trials is increased from 1 to 10 .

There are at least two experimental situations where having this information would be beneficial. The first is where an experiment is conducted simply to demonstrate excitatory conditioning, such as would be the case in a classroom setting. In this situation, one would want to give, for ethical reasons, the smallest number of trials necessary for conditioning to be detected, since this preparation employs an aversive US. The second situation is where the purpose of an experiment is to determine whether a particular treatment has a positive or negative effect on conditioning, relative to controls. In the former, giving too many trials could prevent detection of an actual treatment effect, due to a level of conditioning in controls that is too close to asymptote, whereas in the latter, giving too few trials could produce a level of conditioning in controls that is too low for an actual negative effect to be detected.

\section{Method}

Subjects The subjects were 32 experimentally naïve feeder goldfish (4 to $5 \mathrm{~cm}$ in length) purchased at a local pet store. They were housed in white, plastic buckets filled with about $8 \mathrm{~L}$ of tap water (plus conditioner) and fed the recommended amount of standard goldfish food. Each bucket housed four goldfish, all from the same experimental group. The room temperature was maintained at $21^{\circ} \mathrm{C}$, and a $12 / 12-\mathrm{hr}$ light/ dark cycle was in effect.

Apparatus The essential features of the apparatus are diagrammed in Fig. 1, and its technical specifications were as follows. The conditioning chamber itself consisted of a clear, plastic box $(10.2 \mathrm{~cm} \times 10.2 \mathrm{~cm} \times 12.7 \mathrm{~cm})$ that had a removable square cap $(10.2 \mathrm{~cm} \times 10.2 \mathrm{~cm})$ with a $1.5-\mathrm{cm}$ diameter hole drilled through its center. This chamber was filled, to about $1.5 \mathrm{~cm}$ from the top, with the same type of water used to house the goldfish. A shock-US was produced by connecting a DC power source (Dependable Energy 


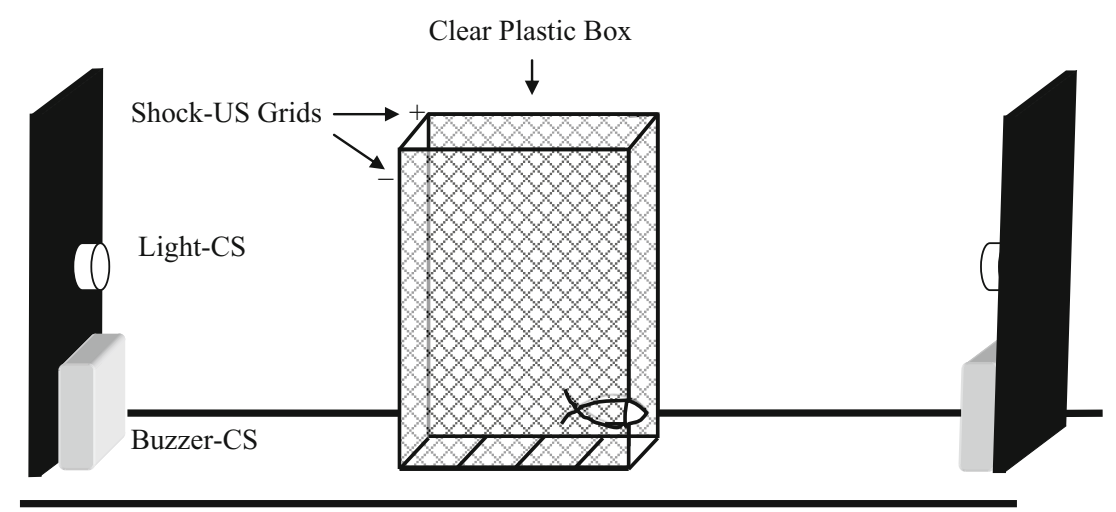

Fig. 1 Goldfish classical conditioning chamber

Brand, Plug-In Class 2 Transformer; Model No: AM-6500) to two shock grids $(10.2 \mathrm{~cm} \times 15.5 \mathrm{~cm})$. These grids were cut from a sheet of galvanized wire mesh and positioned inside the conditioning chamber, with one flush against one wall and connected to the power source's positive terminal, and the other flush against the opposite wall and connected to the power source's negative terminal (the top portion of each grid was folded over the chamber's wall and crimped to secure it in place). When the power source was turned on, electrical current flowed through the water between the two grids, shocking the goldfish as they swam. After having passed through the water, this current measured $10 \mathrm{~V}, 85 \mathrm{~mA}$. To aerate the water in the conditioning chamber, a $0.5-\mathrm{cm}$ rubber air tube was secured (with a piece of electrical wire) flush against, and down the center, of one of the shock grids, with the end of the tube being about $1.5 \mathrm{~cm}$ from the chamber's floor. This tube was connected to a standard aquarium air pump and adjusted to produce one to two bubbles per second. The floor of this chamber was divided into four equal rectangular sections by taking a thin piece of white plastic $(10.2 \mathrm{~cm} \times$ $10.2 \mathrm{~cm}$ ), drawing three straight parallel lines on it, $2.5 \mathrm{~cm}$ apart, with a fine-tipped black marker, and securing it (with a clear adhesive) flush against the outside of the chamber's floor with the black lines facing up and perpendicular to the bottom edges of the two shock grids. The conditioning chamber rested on top of a level, wooden platform, about $1 \mathrm{~m}$ above the room's floor. A visual CS was provided by two round, red pilot lights (6 V DC, $1.27 \mathrm{~cm}$ in diam.), each individually secured to the center of a square piece of thick, black cardboard $(12.7 \mathrm{~cm} \times 12.7 \mathrm{~cm})$. These cardboard pieces were placed in an upright position, $25 \mathrm{~cm}$ away from the conditioning chamber, with one light facing one of the conditioning chamber walls not covered with a shock grid and the other the same distance away from the opposite wall. A small mirror was positioned $18 \mathrm{~cm}$ above the conditioning chamber, at about a $45^{\circ}$ angle, so the goldfish could be viewed by the experimenter, who was sitting at a table about $1 \mathrm{~m}$ from the conditioning chamber. Finally, this conditioning apparatus was located in a room with background sound and illumination levels of $56 \mathrm{~dB}$ and $70 \mathrm{lux}$ (white light), respectively, and maintained at a temperature of $21^{\circ} \mathrm{C}$.

Procedure Subjects were matched in terms of size and color, and then randomly assigned to one of four groups of eight goldfish each. On Day 1, subjects were placed, four at a time, in the conditioning chamber and given either $1,4,7$, or 10 forward delay CS-US trials, with the CS, US, interstimulus interval (ISI; CS onset to US onset) and ITI (trial onset to onset) durations equal to $5,1,4$, and $180 \mathrm{~s}$, respectively. ${ }^{2}$ The first trial began $2 \mathrm{~min}$ and $55 \mathrm{~s}$ into the session, and subjects were returned to their home containers $1 \mathrm{~min}$ after the last trial. On Day 2, subjects were tested, one at a time, for conditioned withdrawal from the CS. This was done by placing the goldfish in the conditioning chamber and, after $3 \mathrm{~min}$, turning on either the left or right CS (in relation to the experimenter) as soon as the goldfish positioned its nose above the left or right end-section of the chamber's floor, respectively, and was not displaying the aforementioned freezing behavior. A handheld timer was started as soon as the CS was turned on, and stopped as soon as the goldfish, withdrawing from the CS, crossed the midline of the floor with its nose. If the midline was crossed in less than $4 \mathrm{~s}$, a conditioned response (CR) was judged to have occurred, and the goldfish was given a $\mathrm{CR}$ percent score of 100; otherwise, the goldfish was given a score of $0 .^{3}$ In all experiments, both training and testing orders were

\footnotetext{
${ }^{2}$ At any given point in time, fish in this chamber are most likely to be oriented toward one of the two walls not covered with wire mesh. Therefore, to ensure perception of the CS during conditioning, both lights were illuminated simultaneously on each trial.

${ }^{3}$ As indicated by Barela (2012), this 4-s CR criterion was chosen to allow for comparisons with potential experiments where conditioning is assessed, not after the completion of training, but rather, on a trial-by-trial basis, during the ISI, which, in the present experiment, was $4 \mathrm{~s}$.
} 
counterbalanced across groups, and the experimenter was blind to the subjects' group membership during CS testing. Also, all stimuli were controlled manually by the experimenter, as in Barela (2012). Finally, trend analyses and/or independent-sample $t$ tests were performed using an SPSS statistical package, with alpha set at .05, two-tailed.

\section{Results and discussion}

Results of the statistical analyses are provided under the Experiment 1A heading in Table 1. Consistent with what has been observed in other preparations, a comparison of the group means indicated that conditioning to the light-CS increased in a direct, negatively accelerated fashion as the number of CS-US trials increased from 1 to 10 . The linear component of this relationship was corroborated by a significant linear trend; however, the curvilinear component was not supported by a significant quadratic trend. Also, replicating what was observed previously in this laboratory, the level of conditioning produced by 10 trials was at an asymptotic level. Finally, to narrow in on the minimum number of trials required to detect conditioning with the methods employed here, pairwise comparisons were made between each group mean and a hypothetical mean of $12.5 \%$, the typical percentage of responses that meet the $\mathrm{CR}$ criterion in nonassociative controls with this preparation (range $=0$ to $25 \%$ ). According to these analyses, seven trials were sufficient to produce a statistically significant level of conditioning, but four were not. $^{4}$

Some practical implications of these results for employing the goldfish conditioned withdrawal preparation are as follows. First, conditioning to the light-CS can be increased in a linear fashion from a control level to asymptote by increasing the number of CS-US trials from 1 to 10 . Second, if the purpose of an experiment is simply to demonstrate conditioning in a two-group controlled study, more than four trials are likely going to be necessary, but seven sufficient, for conditioning to be detected with the methods employed here.

\footnotetext{
${ }^{4}$ During testing, actual latencies to cross the midline of the floor were recorded (maximum $=10 \mathrm{~s}$ ) and then converted to percent CR scores. One reviewer wondered what the results would have looked like with the former used as the dependent variable. Using the data from Experiment $1 \mathrm{~A}$, statistical analyses revealed a strong indirect relationship between percent CRs and latencies, $r=-0.93, p<.001$. In addition, the latter $M s=8.53 \mathrm{~s}, 4.40 \mathrm{~s}, 3.43 \mathrm{~s}$, and $2.58 \mathrm{~s}$, for the 1-, 4-, 7-, and 10 -trial groups, respectively, with the linear trend significant, $F(1,28)=11.27, p=.002$, but the quadratic trend not, $F(1$, $28)=1.71, p=.381$. Thus, the conclusions that can be drawn from these two dependent measures are essentially the same.
}

However, if statistical power is increased by increasing the number of subjects in each group, conditioning can likely be detected with fewer trials than this (see Power Analysis section below). A third implication of these results is that, if an experiment's purpose is to determine whether a particular treatment has a positive or negative effect on conditioning, one to four trials might be optimal in the former case, and 7 to 10 in the latter, for the reasons stated above.

\section{Experiment 1B}

This experiment had two purposes. The first was to replicate the previous finding that seven CS-US trials are sufficient for conditioning to be detected in this preparation, but this time, an actual, rather than a hypothetical, control group was used. The second was to determine whether the original Christmas light-CS used by Barela (2012) is similarly effective in producing conditioning, a determination that would bear on whether the modification made to the CS actually did improve the efficacy of this preparation.

\section{Method}

Subjects The subjects were 32 experimentally naïve feeder goldfish (4 to $5 \mathrm{~cm}$ in length) purchased at a local pet store. They were housed and maintained the same as in Experiment 1A.

Apparatus The apparatus was the same as in Experiment $1 \mathrm{~A}$, except in half the cases (see Procedure) the two red pilot lights (along with the black cardboard squares to which each was affixed) were removed and replaced with two red Christmas lights, each inserted, in an upright position, through a hole $(2.34 \mathrm{~cm}$ in diameter) drilled through the wooden platform, directly below where each pilot light had been located.

Procedure Subjects were matched in terms of size and color, and then randomly assigned to one of four groups of eight goldfish each. On Day 1, subjects were placed in the conditioning chamber and given either seven CS-US trials (Groups Experimental) or seven unpaired presentations of these stimuli (Groups Control) with either the red pilot light- or the red Christmas light-CSs. The procedure employed in the experimental groups was the same as that used in the 7-Trial Group in Experiment 1A. In the control groups, the USs were presented at the same times as those in the experimental groups, but the CSs were presented 1 min earlier. On Day 2, subjects were tested for conditioning to the particular CS they had been trained with, using the same procedure as that in Experiment 1A, which was the standard procedure used throughout. 
Table 1 Statistical analyses of percent conditioned withdrawal responses

\begin{tabular}{|c|c|c|c|c|c|c|}
\hline Group or effect & $M$ & $S D$ & $t$ & $d f$ & $p$ & Cohen's $d$ \\
\hline \multicolumn{7}{|l|}{ Experiment $1 \mathrm{~A}$} \\
\hline 1 trial & 12.50 & 35.36 & & & & \\
\hline 4 trials & 50.00 & 53.45 & & & & \\
\hline 7 trials & 75.00 & 46.29 & & & & \\
\hline 10 trials & 87.50 & 35.36 & & & & \\
\hline Linear trend & & & 3.65 & 28 & $.001^{*}$ & 1.38 \\
\hline Quadratic trend & & & 0.82 & 28 & .421 & \\
\hline$M=12.5$ vs. 1 trial & & & 0.00 & 7 & 1.00 & \\
\hline$M=12.5$ vs. 4 trials & & & 1.98 & 7 & .088 & \\
\hline$M=12.5$ vs. 7 trials & & & 3.82 & 7 & $.007 *$ & 2.89 \\
\hline$M=12.5$ vs. 10 trials & & & 6.00 & 7 & $.001 *$ & 4.54 \\
\hline \multicolumn{7}{|l|}{ Experiment $1 \mathrm{~B}$} \\
\hline \multicolumn{7}{|l|}{ Christmas light-CS } \\
\hline Control & 12.50 & 35.36 & & & & \\
\hline Experimental & 50.00 & 53.45 & & & & \\
\hline Treatment & & & 1.66 & 14 & .120 & \\
\hline \multicolumn{7}{|l|}{ Pilot light-CS } \\
\hline Control & 12.50 & 35.36 & & & & \\
\hline Experimental & 75.00 & 46.29 & & & & \\
\hline Treatment & & & 3.03 & 14 & $.009 *$ & 1.52 \\
\hline \multicolumn{7}{|l|}{ Experiment $1 \mathrm{C}$} \\
\hline Control & 25.00 & 46.29 & & & & \\
\hline Experimental & 75.00 & 46.29 & & & & \\
\hline Treatment & & & 2.16 & 14 & $.049 *$ & 1.08 \\
\hline \multicolumn{7}{|l|}{ Experiment 2} \\
\hline $0 \mathrm{~V}$-shock & 12.50 & 35.36 & & & & \\
\hline 3 V-shock & 25.00 & 46.29 & & & & \\
\hline $6 \mathrm{~V}$-shock & 50.00 & 53.45 & & & & \\
\hline 9 V-shock & 62.50 & 51.75 & & & & \\
\hline Linear trend & & & 2.34 & 28 & $.027 *$ & 0.88 \\
\hline Quadratic trend & & & 0.00 & 28 & 1.00 & \\
\hline 0 vs. 3 V-shock & & & 0.53 & 28 & .601 & \\
\hline 0 vs. 6 V-shock & & & 1.59 & 28 & .124 & \\
\hline 0 vs. 9 V-shock & & & 2.12 & 28 & $.043 *$ & 0.80 \\
\hline \multicolumn{7}{|l|}{ Experiment 3A } \\
\hline Control & 00.00 & 00.00 & & & & \\
\hline Experimental & 50.00 & 53.45 & & & & \\
\hline Treatment & & & 2.65 & 14 & $.019 *$ & 1.42 \\
\hline \multicolumn{7}{|l|}{ Experiment 3B } \\
\hline Control & 25.00 & 46.29 & & & & \\
\hline Experimental & 75.00 & 46.29 & & & & \\
\hline Treatment & & & 2.16 & 14 & $.049 *$ & 1.15 \\
\hline $\begin{array}{l}\text { Experiment 3A vs. 3B } \\
\text { (collapsed) }\end{array}$ & & & 1.46 & 30 & .154 & \\
\hline \multicolumn{7}{|l|}{ Experiment 4} \\
\hline 10-s ITI & 18.18 & 40.45 & & & & \\
\hline 60-s ITI & 50.00 & 52.22 & & & & \\
\hline 300-s ITI & 83.33 & 38.92 & & & & \\
\hline
\end{tabular}

Table 1 (continued)

\begin{tabular}{lllllll}
\hline Group or effect & $M$ & $S D$ & $t$ & $d f$ & $p$ & Cohen's $d$ \\
\hline Linear trend & & & 3.52 & 32 & $.001 *$ & 1.24 \\
$\quad$ Quadratic trend & & & 0.05 & 32 & .962 & \\
Experiment 5 & & & & & & \\
$\quad$ Control & 00.00 & 00.00 & & & & \\
$\quad$ Experimental & 71.43 & 48.80 & & & & \\
$\quad$ Treatment & & & 3.87 & 12 & $.002 *$ & 2.23 \\
\hline
\end{tabular}

Note. Obtained $F$ values were converted to $t$ scores $\left(t^{2}=F\right)$ and $*$ denotes a significant effect

\section{Results and discussion}

Results of the statistical analyses are provided under the Experiment 1B heading in Table 1. It can be seen from these results that, in subjects trained with the red pilot light-CS, the experimental group showed significantly more conditioned withdrawal than the control group, confirming that seven CS-US trials are sufficient for conditioning to be detected in this preparation. However, when subjects were trained with the red Christmas light-CS, this same number of trials failed to produce a significant amount of conditioning, relative to controls. Thus, evidence has now been provided that the modification made to the light-CS did, indeed, improve its efficacy.

\section{Experiment 1C}

As indicated above, the two red Christmas light-CSs used by Barela (2012) were replaced by two red pilot lights, with each of the latter affixed to a black cardboard square. The color black was chosen in an effort to camouflage the bulb, which appeared somewhat dark (due to the bulb recess) when not illuminated. This dark background, along with the relative smallness of the pilot light, seemed to make the latter's illumination more salient than that of the Christmas light, the background of which consisted simply of the room's walls, which were white. The present experiment was conducted to determine whether the darkness of this background is critical to the associability of the red pilot light-CS by examining whether a comparable level of conditioning occurs when the shade of this background is at the opposite end of the grayscale, namely, white.

\section{Method}

Subjects The subjects were 16 experimentally naïve feeder goldfish (4 to $5 \mathrm{~cm}$ in length) purchased at a local pet store. They were housed and maintained the same as in Experiment $1 \mathrm{~A}$. 
Apparatus The apparatus was the same as in Experiment 1A, except that the cardboard square to which the red pilot light was affixed was white instead of black.

Procedure Subjects were matched in terms of size and color, and then randomly assigned to one of two groups of eight goldfish each. On Day 1, goldfish were placed in the conditioning chamber and given either seven CS-US trials (Group Experimental), or seven unpaired presentations of these stimuli (Group Control), as in Experiment 1B. On Day 2, subjects were tested for conditioning to the light-CS using the standard procedure.

\section{Results and discussion}

Results of the statistical analyses are provided under the Experiment $1 \mathrm{C}$ heading in Table 1. It can be seen from these results that seven trials produced a significant amount of conditioning to the light-CS, relative to controls, and that the level of conditioning produced was comparable to that seen in subjects trained in the previous experiments with the black background. Thus, no evidence was provided that the grayscale shade of this background makes a difference with respect to the amount of conditioning that occurs to the red pilot lightCS.

\section{Experiment 2}

In addition to number of CS-US trials, a second methodological variable found to affect classical conditioning is the intensity of the US. In preparations that employ a shock-US, this variable has been found to be directly related to the strength of conditioning produced, an effect observed in both mammals (e.g., Annau \& Kamin, 1961; Kamin \& Brimer, 1963; Morris \& Bouton, 2006) and fish (e.g., Dunlop, Millsopp, \& Laming, 2006; Fukui \& Shishimi, 1985). The present experiment was conducted to see if conditioning can be detected using the methods above, but with a lower shock intensity. If it can, it would be recommended, on ethical grounds, that the lowest effective shock intensity be employed.

\section{Method}

Subjects The subjects were 32 experimentally naïve feeder goldfish (4 to $5 \mathrm{~cm}$ in length) purchased at a local pet store. They were housed and maintained the same as in Experiment 1A.

Apparatus The apparatus was the same as in Experiment 1A, with the exception that the shock-US was provided by a Mastech DC power supply (HY1803D, No. 324978) adjusted to produce an electrical output current of $0,3,6$, or $9 \mathrm{~V}$. After having passed through the water of the conditioning chamber, the nonzero currents measured $3 \mathrm{~V}, 25 \mathrm{~mA} ; 6 \mathrm{~V}, 55 \mathrm{~mA}$; and $9 \mathrm{~V}, 87 \mathrm{~mA}$, respectively. It was readily apparent that the vigor of the thrashing-UR increased as shock intensity increased.

Procedure Subjects were matched in terms of size and color, and then randomly assigned to one of four groups of eight goldfish each. On Day 1, goldfish were placed in the conditioning chamber and given 10 CS-US trials using the same methods as those used in the 10-Trial Group in Experiment $1 \mathrm{~A}$, except one of the above shock intensities was used. On Day 2, subjects were tested for conditioning to the lightCS using the standard procedure.

\section{Results and discussion}

It can be seen, from an examination of the group means under the Experiment 2 heading in Table 1, that there was a direct, linear relationship between conditioning to the light-CS and shock-US intensity, a finding corroborated by a significant linear trend. However, when compared to the group that received no shock, only the $9-\mathrm{V}$ shock-US was able to produce a significant amount of conditioning to this stimulus.

Some practical implications of these results for using the goldfish conditioned withdrawal preparation are as follows. First, conditioning can be increased in a linear fashion by increasing the shock-US intensity from 1 to $9 \mathrm{~V}$, and likely up to $10 \mathrm{~V}$, as well, if the results of Experiment 1A are taken into account. Second, if the purpose of an experiment is simply to demonstrate excitatory conditioning in a two-group, controlled study, with the above methods, a shock-US intensity of $6 \mathrm{~V}$ will likely not be sufficient to obtain a statistically significant effect, but one of $9 \mathrm{~V}$ will. However, if a greater number of trials are going to be given (cf. Barela, 2012) or, again, if statistical power is increased by increasing the number of subjects, one will likely be able to detect conditioning with shock intensities lower than this. Finally, because the 10$\mathrm{V}$ power source used in the previous experiments produced a higher level of conditioning than the $9-\mathrm{V}$ power source used here, using otherwise identical methods (cf. Experiment 1A, 10-Trial Group), the former was used in subsequent experiments.

\section{Experiments 3A and 3B}

A third methodological variable that can affect classical conditioning is the length of the ISI, with this variable having been found to be inversely related to rate of conditioning and positively related to the CS-CR delay, effects seen in both mammals (for a review, see Gallistel \& Gibbon, 2002) and goldfish (Bitterman, 1964; Drew, Couvillon, Zupan, Cooke, 
\& Balsam, 2005). Up to this point, only 4-s ISIs have been used in the goldfish conditioned withdrawal preparation. The present experiments were conducted to determine whether conditioning can be detected with 1- and 9-s ISIs as well. This information could be useful for at least two reasons. First, it could facilitate comparisons with the results of other research, since the most commonly used ISI in previous fear conditioning studies with goldfish has been around 9-10 s. Second, it could aid in the assessment of conditioning to different CSs that are presented sequentially during training, such as in studies on sensory preconditioning, second-order conditioning, and occasion-setting.

\section{Method}

Subjects The subjects were 32 experimentally naïve feeder goldfish (4 to $5 \mathrm{~cm}$ in length) purchased at a local pet store. They were housed and maintained the same as in the above experiments.

Apparatus The apparatus was the same as in Experiment 1A.

Procedure In Experiment 3A, subjects were matched in terms of size and color and then randomly assigned to one of two groups of eight goldfish each. On Day 1, subjects in the experimental group were placed in the conditioning chamber and given 10 CS-US pairings, as in the experiments above, except the duration of the light-CS was $2 \mathrm{~s}$, with a 1$\mathrm{s}$ ISI, and the first trial began $2 \mathrm{~min}$ and $58 \mathrm{~s}$ into the session. In contrast, subjects in the control group were given the same number of CS and US presentations, but unpaired, with the USs occurring at the same times, but the CSs presented $89 \mathrm{~s}$ earlier, than the corresponding stimuli in the experimental group. On Day 2, subjects were tested for conditioning to the CS using the standard procedure, except they had to cross the midline in less than $1 \mathrm{~s}$ for a withdrawal-CR to be counted. In Experiment 3B, this same procedure was used with two new groups of eight goldfish each, except in the experimental group, a 10-s CS with a 9-s ISI was used during training, with the first trial beginning $2 \mathrm{~min}$ and $50 \mathrm{~s}$ into the training session, and in the unpaired control group, the CSs were presented $85 \mathrm{~s}$ earlier than those in the experimental group. As for the CS testing procedure, it was, again, the standard one, except subjects had to cross the midline in less than $9 \mathrm{~s}$ for a withdrawal-CR to be counted.

\section{Results and discussion}

It can be seen from the results presented under the Experiment 3A (1-s ISI) and 3B (9-s ISI) headings in Table 1 that, relative to unpaired controls, a significant level of conditioning was produced by CS-US pairings given with both ISIs. Also, although the overall percentage of withdrawal responses appeared to be lower in the 1-s than in the 9-s ISI groups (collapsed across treatment), this difference was not significant. Thus, it may be concluded that the goldfish conditioned withdrawal preparation can be used to detect conditioning to a light-CS with ISIs ranging from 1 to $9 \mathrm{~s}$.

\section{Experiment 4}

A fourth variable known to affect classical conditioning is the length of the ITI. Specifically, it has been found that conditioning can be facilitated by increasing the length of this interval, a phenomenon referred to as the trial spacing effect (see, e.g., Barela, 1999; Sunsay \& Bouton, 2008; Sunsay, Stetson, $\&$ Bouton, 2004). The purpose of the present experiment was to examine the effect of ITI on conditioning to a light-CS in the goldfish conditioned withdrawal preparation by giving subjects six CS-US trials with ITIs of 10,60 , or $300 \mathrm{~s}$.

\section{Method}

Subjects The subjects were 36 experimentally naïve feeder goldfish (4 to $5 \mathrm{~cm}$ in length) purchased at a local pet store. They were housed and maintained the same as in the above experiments.

Apparatus The apparatus was the same as in Experiment 1A.

Procedure Subjects were matched in terms of size and color and then randomly assigned to one of three groups of 12 goldfish each. On Day 1, subjects were placed in the conditioning chamber and, beginning $2 \mathrm{~min}$ and $55 \mathrm{~s}$ into the session, given six CS-US trials with ITIs of 10,60 , or $300 \mathrm{~s}$. Subjects were returned to their home containers $1 \mathrm{~min}$ after the last trial. On Day 2, subjects were tested for conditioning to the light-CS using the standard procedure.

\section{Results and discussion}

One subject in the 10-s ITI group died. In agreement with what has been found previously in a variety of classical conditioning preparations, it is clear from a comparison of the group means under the Experiment 4 heading in Table 1 that conditioning to the light-CS increased in a linear fashion as the duration of the ITI increased from 10 to $300 \mathrm{~s}$, an observation substantiated by a significant linear, but not quadratic, trend.

Some practical implications of these results for employing the goldfish conditioned withdrawal preparation are as follows. Obviously, one is that, with the methods employed here, one can increase or decrease the level of conditioning produced by increasing or decreasing the ITIs, respectively, across the 10 to $300 \mathrm{~s}$ range. Additionally, it is noteworthy that six trials given with 300 -s ITIs were sufficient to produce 
an asymptotic level of conditioning. Finally, the method used in this experiment could serve as a starting point for future investigations of the trial spacing effect, the underlying mechanisms of which have yet to be fully determined (see, e.g., Barela, 1999; Sunsay \& Bouton, 2008; Sunsay et al., 2004).

\section{Experiment 5}

In the area of classical conditioning, there are many phenomena that require the use of multiple CSs for their study (e.g., sensory preconditioning, second-order conditioning, occasion setting, overshadowing, blocking, and positive and negative patterning). Although the majority of classical conditioning studies with goldfish have employed visual CSs, conditioning to an auditory CS has also been observed using a respiration measure (e.g., Fay, 1970). Up to this point, conditioning in the goldfish conditioned withdrawal preparation has only been directly measured to a visual CS. The present experiment was conducted to determine whether fear conditioning to an auditory CS can be detected with this preparation, as well.

\section{Method}

Subjects The subjects were 16 experimentally naïve feeder goldfish (4 to $5 \mathrm{~cm}$ in length) purchased at a local pet store. They were housed and maintained the same as in the above experiments.

Apparatus The apparatus was the same that used in Experiment 1A, except two small buzzers (Radio Shack, $6 \mathrm{~V} \mathrm{DC}, 78 \mathrm{~dB}, 300$ to $500 \mathrm{~Hz}$ ) served as the CS, with each positioned $2.25 \mathrm{~cm}$ directly under one of the red lights and the speaker facing the conditioning chamber.

Procedure Subjects were matched in terms of size and color and then randomly assigned to one of two groups of eight goldfish each. On Day 1, subjects in the experimental group were placed in the conditioning chamber and given 10 CS-US pairings, as in Experiment 1A. In contrast, subjects in the control group were given the same number of CS and US presentations, but in an unpaired fashion; specifically, the USs occurred at the same times as those in the experimental group, but the 5-s buzzer-CS occurred $90 \mathrm{~s}$ earlier. Unlike the two red lights in the above experiments, which were turned on simultaneously during training, only one buzzer was turned on at a time, one on odd-numbered trials, and the other on even-numbered ones. On Day 2, subjects were tested for conditioning to the buzzer-CS using the standard procedure.

\section{Results and discussion}

Two subjects (one in each group) died. It can be seen from the results presented under the Experiment 5 heading in Table 1 that the subjects in the experimental group showed significantly more conditioning to the buzzer-CS than those in the control group. Thus, it may be concluded that the goldfish conditioned withdrawal preparation can be used to detect conditioning, not only to a visual CS, but to an auditory one as well.

\section{Power analysis}

After collecting the above data, a power analysis was conducted using $G^{*}$ Power 3.1.9.2 for Windows (Faul, Erdfelder, Lang, \& Buchner, 2007), which can be downloaded from the internet for free, to help future employers of this preparation estimate the minimum number of subjects needed to detect small, medium and large treatment effects when using a two-group, independent sample $t$ test with alpha $=0.05$ (twotailed), power $=0.80$, and Cohen's $d$ as a measure of effect size, where $d=M_{1}-M_{2} / \sigma$. In the present report, the largest treatment effect was a group difference $\left(M_{1}-M_{2}\right)=75 \%$. Thus, small, medium, and large treatment effects could reasonably be defined as group means differing by $25 \%, 50 \%$, and $75 \%$, which, using a value of $\sigma=41.51$ (weighted average of the $S D s$ [population estimates] in Table 1), would correspond to Cohen's $d$ values of $0.60,1.20$, and 1.81 , respectively. Using the above parameter values, it was determined that a minimum of 45,12 , and 6 subjects per group would be necessary to detect small, medium, and large treatment effects, respectively, with this preparation.

\section{General discussion}

The research reported here was conducted to (1) provide empirical data that can help employers of the goldfish conditioned withdrawal preparation determine the optimal experimental methods for their purposes, and (2) determine whether some of the modifications made to the original preparation were, indeed, improvements. Consistent with what has been found previously in a wide variety of classical conditioning preparations and animal subjects, the results showed that conditioning to a light-CS was directly related to the number of CS-US trials, intensity of the US, and duration of the ITI. In addition, conditioning was detected with ISIs slightly longer and shorter than those used previously, as well as with an auditory CS. Finally, evidence was provided that the pilot lights were more effective than the original Christmas lights in producing conditioning. Thus, this research advances the development of a low-cost preparation for the study of classical conditioning in vertebrates, and provides basic 
methodological data that can expedite its use, both in the classroom and in professional research laboratories.

Although other preparations have successfully employed goldfish in the study of classical fear conditioning, the one described in this report might have some comparative advantages. For example, preparations that have used general activity (e.g., Bitterman, 1964), respiration (e.g., Fay, 1970; Xu, 1997), and heart-rate (e.g., Yoshida, Okamura, \& Uematsu, 2004) to index conditioning seem to be much more technically sophisticated, and therefore, more expensive and difficult for the novice to construct from scratch than the one described here. Although the shuttle-box preparation (which was originally designed to study avoidance learning but can also be used to study classical conditioning) might be similar to the present one in terms of cost and ease of construction, the former appears to require many more trials for conditioning to be detected (cf. Woodard \& Bitterman, 1971). Finally, the goldfish conditioned withdrawal preparation now has a broader base of methodological data from which to draw when planning classroom demonstrations or designing research experiments.

As indicated previously (Barela, 2012), although this preparation has many positive attributes, it has at least one major shortcoming: it is not automated. Because of this, conducting experiments can be very tedious and time-consuming for the experimenter. Also, because of the human factors involved, there is an increased chance of procedural errors being made. To remedy this problem, Barela is currently in the process of automating this preparation using the microcontroller/computer programming methods described by Varnon and Abramson (2013), which are neither too difficult nor expensive (less than \$150) for the novice. However, as one reviewer of this paper pointed out regarding this preparation's use in the classroom, "students learn more from manually carrying out conditioning procedures than from simply turning on a switch and watching how the apparatus works."

\section{References}

Annau, Z., \& Kamin, L. J. (1961). The conditioned emotional response as a function of the intensity of the US. Journal of Comparative and Physiological Psychology, 54, 428-432.

Barela, P. B. (1999). Theoretical mechanisms underlying the trial spacing effect in Pavlovian fear conditioning. Journal of Experimental Psychology: Animal Behavior Processes, 25, 177-193.

Barela, P. B. (2012). Conditioned withdrawal in goldfish: A simple and inexpensive preparation for the study of fear conditioning in vertebrates. Psychological Reports, 110, 173-186.

Bitterman, M. E. (1964). Classical conditioning in the goldfish as a function of the CS-US interval. Journal of Comparative and Physiological Psychology, 58, 359-366.
Cohen, J. (1988). Statistical power analysis for the behavioral sciences (2nd ed.). Hillsdale: Erlbaum.

Drew, M. R., Couvillon, P. A., Zupan, B., Cooke, A., \& Balsam, P. D. (2005). Temporal control of conditioned responding in goldfish. Journal of Experimental Psychology: Animal Behavior Processes, 31, 31-39.

Dunlop, R., Millsopp, S., \& Laming, P. (2006). Avoidance learning in goldfish (Carassius auratus) and trout (Oncorhynchus mykiss) and implications for pain perception. Applied Animal Behavior Science, 97, 255-271.

Faul, F., Erdfelder, E., Lang, A. G., \& Buchner, A. (2007). G*Power 3: A flexible statistical power analysis program for the social, behavioral, and biomedical sciences. Behavior Research Methods, 39, 175-191.

Fay, R. R. (1970). Auditory frequency generalization in the goldfish (Carassius auratus). Journal of the Experimental Analysis of Behavior, 14, 353-360.

Fukui, H., \& Shishimi, A. (1985). Effects of US and CS intensities on classical conditioning in goldfish (Carassius auratus). Annual of Animal Psychology, 34, 75-85.

Gallistel, C. R., \& Gibbon, J. (2002). The symbolic foundations of conditioned behavior. Hillsdale: Erlbaum.

Gallistel, C. R., Fairhurst, S., \& Balsam, P. (2004). The learning curve: Implications of a quantitative analysis. Proceedings of the National Academy of Sciences of the United States of America, 101, 13124 13131.

Holland, P. C. (1977). Conditioned stimulus as a determinant of the form of the Pavlovian conditioned response. Journal of Experimental Psychology: Animal Behavior Processes, 3, 77-104.

Kamin, L. J., \& Brimer, C. J. (1963). The effects of intensity of conditioned and unconditioned stimuli on a conditioned emotional response. Canadian Journal of Psychology, 17, 194-198.

Mackintosh, N. J. (1974). The psychology of animal learning. London: Academic Press.

Miskovsky, C., Becker, B., Hilker, A., \& Abramson, C. I. (2010). The fish stick: An easy-to-use classroom training apparatus for fish. Psychological Reports, 106, 135-146.

Morris, R. W., \& Bouton, M. E. (2006). Effect of unconditioned stimulus magnitude on the emergence of conditioned responding. Journal of Experimental Psychology: Animal Behavior Processes, 32, 371385.

Phelps, B. J. (2014). Operant rodent manipulanda applied with goldfish. Innovative Teaching, $3,8$.

Skedsvold, P. (2015, February 9). The US president's fiscal year 2016 budget and what it means for research. Retrieved from http://www. psychonomic.org/news-detail/he-us-president-s-fiscal-year-2016budget-what-it

Sunsay, C., \& Bouton, M. E. (2008). Analysis of a trial-spacing effect with relatively long intertrial intervals. Learning \& Behavior, 36, 104-115.

Sunsay, C., Stetson, L., \& Bouton, M. E. (2004). Memory priming and trial spacing effects in Pavlovian learning. Learning \& Behavior, 32, 220-229.

Varnon, C. A., \& Abramson, C. I. (2013). The propeller experiment controller: Low-cost automation for classroom experiments in learning and behavior. Innovative Teaching, 2, 2.

Woodard, W. T., \& Bitterman, M. E. (1971). Classical conditioning of goldfish in the shuttle box. Behavior Research Methods and Instrumentation, 3, 193-194.

Xu, X. (1997). NMDA receptor antagonist MK-801 selectively impairs learning of the contiguity of the conditioned stimulus and unconditioned stimulus in goldfish. Pharmacology Biochemistry \& Behavior, 58, 491-496.

Yoshida, M., Okamura, I., \& Uematsu, K. (2004). Involvement of the cerebellum in classical fear conditioning in goldfish. Behavioral Brain Research, 153, 143-148. 